\title{
Ahmed glaucoma valve in uveitic patients with fluocinolone acetonide implant-induced glaucoma: 3-year follow-up
}

This article was published in the following Dove Press journal: Clinical Ophthalmology

\author{
Buraa Kubaisi, ${ }^{1,2}$ \\ Arash Maleki ${ }^{1,2}$ \\ Aseef Ahmed 1,2 \\ Neel Lamba ${ }^{1,2}$ \\ Haitham Sahawneh ${ }^{1,2}$ \\ Andrew Stephenson ${ }^{1,2}$ \\ Alyssa Montieth ${ }^{1,2}$ \\ Shobha Topgi ${ }^{3}$ \\ C Stephen Foster ${ }^{1,2,4}$
}

'Massachusetts Eye Research and Surgery Institution, Waltham, MA, USA; ${ }^{2}$ Ocular Immunology \& Uveitis Foundation, Waltham, MA, USA; ${ }^{3}$ The State University of New York Downstate, Brooklyn, NY, USA; ${ }^{4}$ Harvard Medical School, Boston, MA, USA
Correspondence: C Stephen Foster Massachusetts Eye Research and Surgery Institution, 1440 Main Street, Suite 20I, Waltham, MA 0245I, USA

Tel + I 78I 89I 6377

Fax +| 78| 647 |430

Email sfoster@mersi.com
Purpose: To evaluate the efficacy and safety of Ahmed glaucoma valve (AGV) in eyes with noninfectious uveitis that had fluocinolone acetonide intravitreal implant (Retisert ${ }^{\mathrm{TM}}$ )-induced glaucoma.

Methods: This retrospective study reviewed the safety and efficacy of AGV implantation in patients with persistently elevated intraocular pressure (IOP) after implantation of a fluocinolone acetonide intravitreal implant at the Massachusetts Eye Research and Surgery Institution between August 2006 and November 2015.

Results: Nine patients with 10 uveitic eyes were included in this study, none of which had preexisting glaucoma in the study eye. Mean patient age was 42 years; 6 patients were female and 3 were male. Baseline mean IOP was $30.6 \mathrm{mmHg}$ prior to AGV placement while mean IOP-lowering medications were 2.9. In the treatment groups, there was a statistically significant reduction in post-AGV IOP. IOP was lowest at 1-week after AGV implantation $(9.0 \mathrm{mmHg})$. Nine out of 10 eyes achieved an IOP below target value of $22 \mathrm{mmHg}$ and/or a $20 \%$ reduction in IOP from baseline 1 month and 1 year following AGV placement. All other postoperative time points showed all 10 eyes reaching this goal. A statistically significant decrease in IOP-lowering medication was seen at the 1-week, 1-month, and 3-year time points compared to baseline, while a statistically significant increase was seen at the 3-month, 6-month, and 2-year post-AGV time points. No significant change in retinal nerve thickness or visual field analysis was found.

Conclusion: AGV is an effective and safe method of treatment in fluocinolone acetonide intravitreal implant-induced glaucoma. High survival rate is expected for at least 3 years.

Keywords: uveitis, glaucoma, Ahmed glaucoma valve, fluocinolone acetonide intravitreal implant, ocular inflammation

\section{Introduction}

Uveitis is a condition that involves inflammation of the uveal tract or adjacent ocular structures and can lead to severe vision loss if not treated appropriately and effectively. The goal of treatment is to prevent damage to ocular structures by safely and effectively eliminating inflammation. Topical, intravitreal, and periocular corticosteroids have been a mainstay of treatment for uveitis, but their effects have been found to be short acting and transient in efficacy. Systemic corticosteroids, although highly efficacious in treating systemic and ocular inflammation, are associated with numerous systemic side effects with prolonged use. Fluocinolone acetonide intravitreal implant (Retisert ${ }^{\mathrm{TM}}$ ) has been approved by the United States Food and Drug Administration for the treatment of chronic noninfectious uveitis. It is a good therapeutic alternative for patients for whom systemic immunomodulatory therapy is not tolerated, is not effective, 
or is contraindicated. The implant achieves high concentrations in the eye with very low systemic absorption and remains effective for up to 3 years. ${ }^{1}$ However, the fluocinolone acetonide intravitreal implant carries the risks associated with chronic ocular exposure to steroids, primarily cataract formation and elevated intraocular pressure (IOP). The increased incidence of elevated IOP leading to glaucomatous damage in patients who have received the fluocinolone acetonide intravitreal implant has led many patients to require the use of topical hypotensive ocular medications, laser trabeculoplasty, or filtering surgery. ${ }^{2-4}$

Glaucoma drainage implants have become an important method of controlling IOP by shunting aqueous humor from the anterior chamber via a tube into a bleb overlying the plate or band implant that is located at the equatorial subconjunctival space. The aqueous humor filters through the bleb and is reabsorbed into the ocular and systemic circulation, lowering the IOP. Several drainage devices are available in the United States with different sizes, shapes, materials, and the option of a flow-limiting valve as well. The most commonly used valved implant in the United States is the Ahmed glaucoma valve (AGV), which consists of a silicone tube connected to a silicone sheet valve held in a polypropylene body. The valve mechanism consists of 2 thin silicone elastomer membranes. The valved design has been found to reduce the incidence of postoperative hypotony. ${ }^{5-7}$

This study evaluated the safety and efficacy of the AGV in eyes with elevated IOP, presumed to be secondary to the previous placement of a fluocinolone acetonide intravitreal implant.

\section{Materials and methods}

Approval for this study was obtained through the New England Institutional Review Board, which issued a waiver of informed consent based on standard operating procedures for retrospective chart reviews. This study was performed in accordance with the Declaration of Helsinki and was compliant with the Health Insurance Portability and Accountability Act.

This study is a single-center study that was done at the Massachusetts Eye Research and Surgery Institution. It is a retrospective case series of patients with uveitis who were treated with placement of a fluocinolone acetonide intravitreal implant, developed uncontrolled elevated IOP, and subsequently underwent AGV implantation, all between August 2006 and November 2015.

All patients with fluocinolone acetonide intravitreal implant glaucoma and Ahmed valve shunt procedure with at least 3-year follow-up between August 2006 and
November 2015 were included in the study. This group of patients was further screened to identify those who were on active follow-up 3 or more years post-AGV implantation and had no previous history of treatment for elevated IOP. Patients who developed steroid-related elevated IOP in this group were included in the study. Only medical records of the patients who developed elevated IOP were retrospectively reviewed. Elevated IOP was defined as $21 \mathrm{mmHg}$ or above following the surgery.

Based on the chart review, data of patients who developed elevated IOP following fluocinolone acetonide intravitreal implant surgery were collected and reviewed. This included demographic information, clinical history, and pertinent operative/preoperative data. In addition, treatments and treatment outcomes were also noted. All complications postAGV implant surgery were recorded.

All AGV surgeries were done in a similar fashion. All patients had undergone Retisert implantation previously; therefore, AGV was performed on the superior quadrant for each eye. All patients were prepped and draped in the usual sterile fashion for ophthalmic surgery, and lid speculum was then used to obtain exposure. A 7.0 Vicryl suture at the perilimbal cornea was used to obtain further exposure. A conjunctival peritomy was performed with a size of 4-5 clock hours. The subtenon's pocket was opened afterward using the Steven's iris scissors. Some patients needed cautery to obtain hemostasis in the area for the valve placement. A $9.0 \mathrm{~mm}$ distance was marked from the limbus, and the Ahmed valve was then brought to the operative field and primed with balanced salt solution cannula. The valve was placed in a subtenon's pocket and sutured into place with 8.0 Nylon sutures at the premarked area. Tube was then placed on the ocular surface and a distance of $2.0 \mathrm{~mm}$ anterior to the limbus was marked. The tube was trimmed in beveled fashion using Vannas scissors. Paracentesis wound was then created and the anterior chamber was deepened with BCC. Using a 23-gauge bent butterfly needle, the anterior chamber was entered $2.0 \mathrm{~mm}$ posterior to the limbus in planar with the iris. The tube was then inserted into the anterior chamber. After satisfaction with the tube placement, the tube was sutured down with 8.0 Nylon suture in mattress style fashion. A scleral transplant was then placed on top of the tube and sutured down with 8.0 Vicryl sutures. The conjunctiva was then closed with 8.0 Vicryl sutures in interrupted fashion. At the end of the surgery, all patients received Tobradex ointment on the ocular surface and the eye was patched until the next morning.

IOP was measured at all visits using Goldmann applanation tonometry. Any medications used to control IOP were 
recorded at all visits. Best-corrected visual acuity was measured with a Snellen chart at all visits.

The optic nerve was evaluated with retinal nerve fiber layer-optical coherence tomography (rNFL-OCT) on the spectral-domain OCT machine RTVue (Optovue Inc., Fremont, CA, USA) model-RT100 with algorithm version (6.11.0.12). Visual field testing was performed and evaluated using the 24-2 Swedish Interactive Threshold Activity fast (SITA-fast) Humphrey Visual Field on the Humphrey Visual Field Analyzer (Carl Zeiss Meditec, Inc., Dublin, CA, USA). Results from testing of the visual field and rNFL-OCT of the study eye were recorded prior to AGV implantation. All patients had rNFL-OCT at least every 6 months and visual field testing at least once a year to monitor for progression.

\section{Statistical analysis}

The results for each group for all ocular measurements were recorded as mean \pm the standard error of the mean. Significant differences in outcomes were determined by one-way analysis of variance (ANOVA) with Tukey-Kramer multiple comparison a posteriori analysis. All data were analyzed using the Minitab ${ }^{\circledR}$ Statistical Software (Minitab Inc., State College, PA, USA), where a $p$-value $<0.05$ was considered statistically significant.

\section{Results}

\section{Baseline characteristics}

A total of 9 patients and 10 uveitic eyes that underwent postRetisert AGV placement were included in this study. Four patients were diagnosed with panuveitis, 2 with Birdshot retinochoroidopathy, 1 with juvenile idiopathic arthritis associated uveitis, 1 with retinal vasculitis, and 1 with ulcerative colitis-associated uveitis. None of the patients had preexisting glaucoma in their study eye(s). The mean age of our patients was $42 \pm 12.4$ years (range $23-57$ ). Of our 9 patients, 6 were female (Table 1$)$. Three eyes $(30 \%)$ had a visual acuity below

Table I Patient background and history

\begin{tabular}{|c|c|c|c|}
\hline $\begin{array}{l}\text { Case } \\
\text { number }\end{array}$ & $\begin{array}{l}\text { Agel } \\
\text { gender }\end{array}$ & $\begin{array}{l}\text { Study } \\
\text { eye }\end{array}$ & Type of uveitis \\
\hline I & $53 / F$ & OS & Retinal vasculitis \\
\hline 2 & $44 / M$ & OS & Idiopathic panuveitis $\mathrm{OU}$ \\
\hline 3 & $57 / F$ & OD & Ulcerative colitis, uveitis \\
\hline 4 & $37 / F$ & OD, OS & Panuveitis OU, NOD 2 gene defect \\
\hline 5 & $25 / F$ & OD & Idiopathic panuveitis OU; HLA-DRI5+ \\
\hline 6 & $55 / M$ & OD & Birdshot retinochoroidopathy \\
\hline 7 & $47 / M$ & OS & Birdshot retinochoroidopathy \\
\hline 8 & $37 / F$ & OS & Idiopathic panuveitis \\
\hline 9 & $23 / F$ & OD & JIA-associated uveitis \\
\hline
\end{tabular}

Abbreviations: $F$, female; JIA, juvenile idiopathic arthritis; $M$, male.
20/100 prior to AGV placement. At baseline, mean IOP was $30.6 \pm 3.3 \mathrm{mmHg}$ and mean IOP-lowering medications

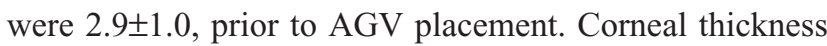
at the pre-Ahmed valve time point was determined using a pachymeter, which revealed an average of $522.5 \mu \mathrm{m}$, with use of available data. The mean duration of time between Retisert implantation and AGV placement was 74 weeks.

\section{Postoperative IOP}

The IOP was determined at the following time points: preRetisert implantation, pre-AGV placement, and 1-week, 1-, 3-, 6-month, 1-, 2-, and 3-year post-AGV. One-way ANOVA revealed a statistically significant change in IOP among the treatment groups $(p<0.05)$. Multiple comparisons with Tukey-Kramer post hoc test revealed that the mean IOP at the pre-AGV time point ("baseline") was significantly higher compared to all other time points $(p<0.001)$ (Figure 1$)$. The IOP was lowest at 1 week following AVG implantation $(9.0 \pm 1.6 \mathrm{mmHg})$, with an insignificant rise at 1-month post AGV (16.9 \pm 1.6$)$, and gradual decline in the following months and years. Of note, 9 eyes (90\%) achieved an IOP below a target threshold of $22 \mathrm{mmHg}$ and/or a $20 \%$ or more reduction in IOP in comparison to the pre-AGV time point, at the 1-month and 1-year time points. All 10 eyes reached this goal for all other time points. Both criteria were met at all follow-up time points in 5 study eyes (50\%) from 5 patients.

\section{Postoperative medications}

The mean number of IOP-reducing drops for the study eyes was determined at the same time points as IOP, starting with

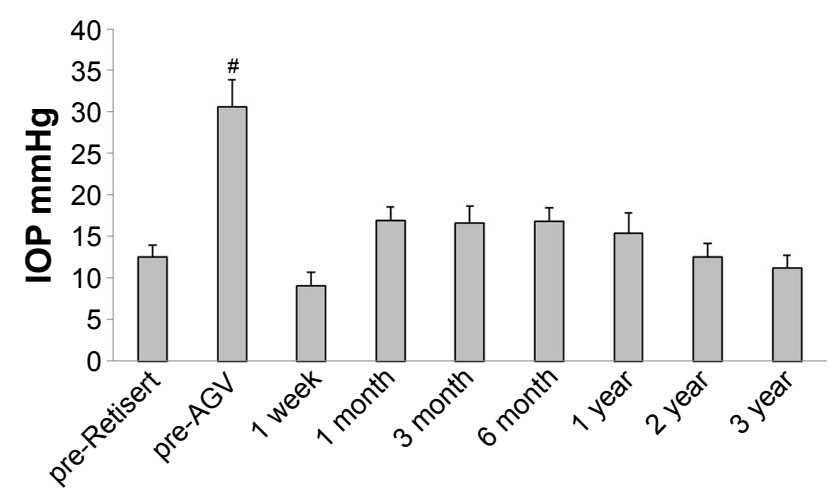

Figure I Mean IOP.

Notes: The IOP for the study eyes was determined at the pre-Retisert, pre-Ahmed valve, and I-week, I-, 3-, and 6-month and I-, 2-, and 3-year follow-up time points. Results for each group are represented as mean \pm SEM. \#Indicates values that are significantly different from all other time points, as assessed by one-way ANOVA with Tukey-Kramer post hoc test $(p<0.05)$.

Abbreviations: AGV, Ahmed glaucoma valve; ANOVA, analysis of variance; IOP, intraocular pressure. 
pre-AGV. One-way ANOVA with Tukey-Kramer post hoc test revealed a statistically significant change in IOP-reducing medication, with a significant decrease in medication at 1 -week $(0.4 \pm 0.3)$ and 1 -month $(0.5 \pm 0.3)$ post-AGV implantation when compared to pre-AGV $(2.9 \pm 0.1)$ (Figure 2). This was followed by a significant rise in IOP medication use at 3-month (1.9 \pm 0.2$), 6$-month (2.2 \pm 0.3$)$, and 2-year $(1.9 \pm 0.4)$ post-AGV compared to the 1-week and 1-month post-AGV. Multiple comparisons also revealed a significant rise in IOP medication use at the 1-year time point $(1.8 \pm 0.4)$ when compared to 1 month. Finally, the mean IOP-lowering medication use at the 3 -year post-AGV time point $(1.4 \pm 0.3)$ was significantly reduced when compared to the pre-AGV time point. The number of patients on oral acetazolamide gradually increased then decreased with the chronological time points. Starting with the pre-AGV period, the number of patients on oral acetazolamide was 8, 0, 0, 1, 2, 4, 2, and 2 .

\section{Visual acuity changes}

Three eyes (30\%) had a visual acuity below a 20/100 cutoff at the pre-Retisert time point. This proportion was the same at the pre-AGV point and then dropped to $2(20 \%)$ at the 1-, $2-$, and 3-year time points. An analysis of the OCT of the rNFL was also performed. One-way ANOVA revealed a lack of significant change in nerve thickness among the various time points $(p>0.05)$. This was confirmed by Tukey-Kramer post hoc test (Figure 3). Finally, an analysis of the Humphrey visual field among the various time points using ANOVA and Tukey-Kramer post hoc test revealed no statistically

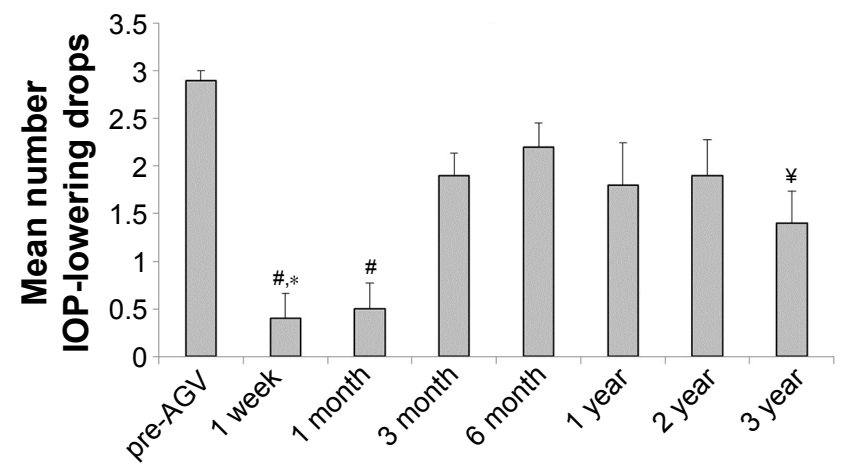

Figure 2 Mean number of IOP-lowering medications.

Notes: The mean number of IOP-lowering drops used for the study eyes was determined at the pre-AGV, I-week, I-, 3-, and 6-month, and I-, 2-, and 3-year follow-up time points. Results for each group are represented as mean \pm SEM. Differences between means were assessed by one-way ANOVA with Tukey-Kramer post hoc test $(p<0.05)$. "Indicates significant difference from pre-AGV, 3-month, 6-month, and 2-year time points. *Indicates significant difference from I year group. ¥Indicates significant difference from pre-AGV time point.

Abbreviations: AGV, Ahmed glaucoma valve; ANOVA, analysis of variance; IOP, intraocular pressure; SEM, standard error of the mean.

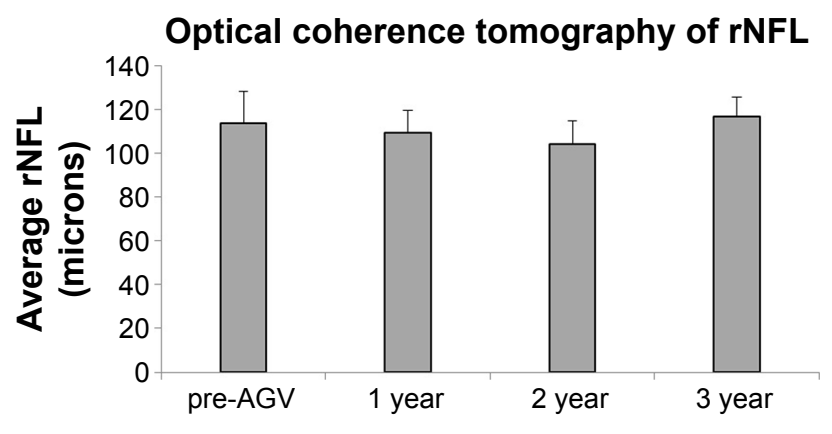

Figure 3 rNFL thickness.

Notes: The rNFL thickness for the study eyes as determined by OCT was assessed at the pre-Ahmed valve and I-, 2-, and 3-year follow-up time points. Results for each group are represented as mean \pm SEM. Significance was determined by one-way ANOVA with Tukey-Kramer post hoc test $(p<0.05)$.

Abbreviations: AGV, Ahmed glaucoma valve; ANOVA, analysis of variance; rNFL, retinal nerve fiber layer; OCT, optical coherence tomography; SEM, standard error of the mean.

significant change for either the mean deviation or the pattern standard deviation $(p>0.05)$ (Figure 4). The mean deviation and pattern standard deviation were both stable by 3 -year follow-up.

\section{Safety of procedure}

Safety was assessed in all patients included in the study. None of the patients developed side effects immediately following Retisert implantation or AGV placement or at time of followups. One patient did require a valve revision. With regard to other ocular complications and/or limitations, 1 patient required selective laser trabeculoplasty at 1-year post-AGV placement due to persistent use of pressure reducing drops. This was followed by trabeculectomy with mitomycin C and laser suture lysis. Another patient had the Retisert removed after 5 years and developed retinal detachment, which was

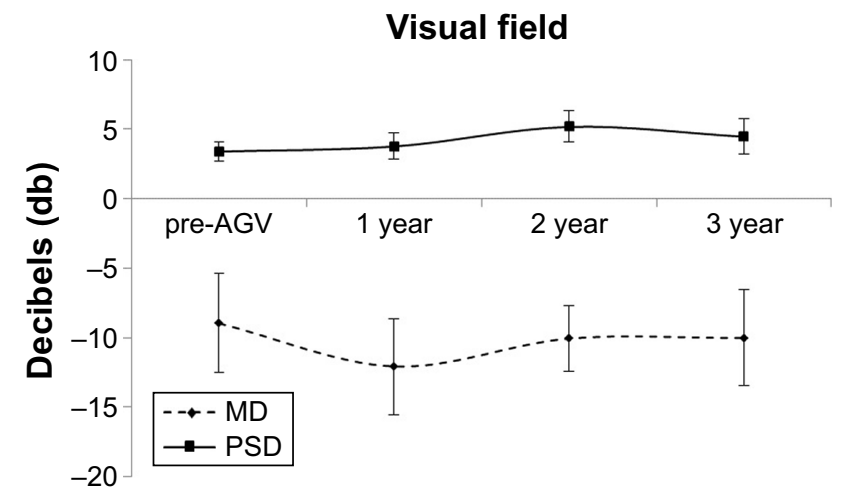

Figure 4 Humphrey Visual Field.

Notes: Analysis of the visual field was made by observation of the MD and PSD. These were assessed at the pre-Ahmed valve, and I-, 2-, and 3-year follow-up time points. Results for each group are represented as mean \pm SEM. Significance was determined by one-way ANOVA with Tukey-Kramer post hoc test $(p<0.05)$.

Abbreviations: AGV, Ahmed glaucoma valve; ANOVA, analysis of variance; MD, mean deviation; SEM, standard error of the mean; PSD, pattern standard deviation. 
repaired with a scleral buckle and silicone oil. Yet another patient required an Ex-PRESS shunt surgery at 1-year postAhmed valve placement, and IOP was stable after this procedure. Finally, 1 patient had poor visual fixation, limiting the number of available results from testing.

\section{Discussion}

Elevated IOP has been found as a consequence of all methods of administration of coritcosteroids and the sustained release of corticosteroids from the fluocinolone acetonide intravitreal implant can lead to elevated IOP that is often difficult to control. Notably, studies have shown that $32 \%-40 \%$ of patients with fluocinolone acetonide intravitreal implant eventually require glaucoma surgery. ${ }^{8,9}$

Selective laser trabeculoplasty has been studied in the treatment of steroid-induced glaucoma in uveitic patients with moderate rates of success. ${ }^{10}$

The corticosteroid response rate has been shown to increase dramatically in eyes where it is believed the conventional aqueous outflow pathway may already be compromised. Similarly to eyes with preexisting primary open-angle glaucoma, eyes with a history of uveitis may have an increased outflow resistance and thus are at a high-risk for corticosteroid-induced elevated IOP. ${ }^{11-13}$

Few retrospective case series have studied the combined AGV and fluocinolone acetonide intravitreal implant in the treatment of uveitic glaucoma. ${ }^{11,12}$

Most of the patients in the Zivney et $\mathrm{al}^{11}$ study underwent sequential surgery, with first fluocinolone acetonide intravitreal implant and then AGV insertion. In contrast, Moore et $\mathrm{al}^{12}$ studied patients who needed combined surgery. In these 2 studies, they did not differentiate between uveitis-induced glaucoma and steroid-induced glaucoma, which is also common in uveitic patients and thus may be a source of bias.

In this study, we evaluated the safety and efficacy of sequential fluocinolone acetonide intravitreal implant and $\mathrm{AGV}$ in the treatment of steroid-induced glaucoma in uveitic patients with long follow-up periods.

Our success rate was the same as the Moore et $\mathrm{al}^{12}$ Study, considering the longer follow-up period. This high success rate indicates 2 hypotheses: 1) good response of steroidinduced glaucoma to AGV even in uveitic eyes and 2) better control of inflammation with fluocinolone acetonide implant can increase success rate. However, previous studies do not support the second hypothesis. ${ }^{11,12}$

A significant decrease in IOP and number of medications after AGV suggests that AGV can be a good option for uveitic patients with steroid-induced glaucoma. Moreover, low rate of early and late complications indicates that AGV can be provided to the patient as an option soon after the development of glaucoma as high numbers of patients eventually need surgical procedures.

The significant increase in topical IOP-lowering medication 3 months after AGV implantation is perhaps the result of a delayed hypertensive episode or a change in the specific eye drops at this time. An increase at this time interval has been shown in previous studies. ${ }^{12,13}$

Two patients who failed AGV were successfully treated with selective laser trabeculoplasty and mini shunt. This demonstrates that good control of inflammation potentially increases the success rate of other procedures in uveitic patients. However, this hypothesis should be proven with more robust studies.

Despite the long follow-up period as a strength point, our study was limited by retrospective design and low number of patients.

\section{Conclusion}

$\mathrm{AGV}$ is an effective and safe method of treatment in fluocinolone acetonide intravitreal implant-induced glaucoma. High survival rate is expected for at least 3 years. However, a randomized clinical trial is necessary to compare the results of sequential and combined fluocinolone acetonide intravitreal implant and AGV insertion. Moreover, a randomized clinical trial could compare mini shunt with $\mathrm{AGV}$ as a less invasive procedure.

\section{Acknowledgments}

Minitab $^{\circledR}$ and all other trademarks and logos for the Company's products and services are the exclusive property of Minitab Inc. All other marks referenced remain the property of their respective owners. See minitab.com for more information. The organization had no role in the design or conduct of this research.

\section{Disclosure}

C Stephen Foster has the following financial disclosures: consultancies with Aldeyra Therapeutics (Lexington, MA, USA), Bausch \& Lomb Surgical, Inc. (Rancho Cucamonga, CA, USA), Eyegate Pharma (Waltham, MA, USA), Novartis (Cambridge, MA, USA), pSivida (Watertown, MA, USA), and Xoma (Berkeley, CA, USA); grants or grants pending with Alcon (Aliso Viejo, CA, USA), Aldeyra Therapeutics (Lexington, MA, USA), Bausch \& Lomb (Bridgewater, NJ, USA), Clearside Biomedical (Alpharetta, GA, USA), Dompé pharmaceutical (Milan, Italy), Eyegate Pharma (Waltham, MA, USA), Icon (Dublin, Ireland), Novartis Pharmaceuticals 
(Cambridge, MA, USA), pSivida (Watertown, MA, USA), Santen (Osaka, Japan), Xoma (Berkeley, CA, USA); payment for lectures including service on speaking bureaus: Alcon (Aliso Viejo, CA, USA), Allergan (Dublin, Ireland); and stock or stock options: Eyegate Pharma (Waltham, MA, USA). The authors report no other conflicts of interest in this work.

\section{References}

1. Driot JY, Novack GD, Rittenhouse KD, Milazzo C, Pearson PA. Ocular pharmacokinetics of fluocinolone acetonide after Retisert intravitreal implantation in rabbits over a 1-year period. J Ocul Pharmacol Ther. 2004;20(3):269-275.

2. Arcinue CA, Ceron OM, Foster CS. A comparison between the fluocinolone acetonide (Retisert) and dexamethasone (Ozurdex) intravitreal implants in uveitis. J Ocul Pharmacol Ther. 2013;29(5):501-507.

3. Callanan DG, Jaffe GJ, Martin DF, Pearson PA, Comstock TL. Treatment of posterior uveitis with a fluocinolone acetonide implant: three-year clinical trial results. Arch Ophthalmol. 2008;126(9):1191-1201.

4. Friedman DS, Holbrook JT, Ansari H, et al. Risk of elevated intraocular pressure and glaucoma in patients with uveitis: results of the multicenter uveitis steroid treatment trial. Ophthalmology. 2013;120(8): 1571-1579.

5. Papadaki TG, Zacharopoulos IP, Pasquale LR, Christen WB, Netland PA, Foster CS. Long-term results of Ahmed glaucoma valve implantation for uveitic glaucoma. Am J Ophthalmol. 2007;144(1):62-69.
6. Da Mata A, Burk S, Netland PA, Baltatzis S, Christen W, Foster CS. Management of uveitic glaucoma with the use of Ahmed valve. Ophthalmology. 1999;106:2168-2172.

7. Ozdal PC, Vianna RNG, Deschenes J. Ahmed valve implantation in glaucoma secondary to chronic uveitis. Eye. 2006;20(2):178-183.

8. Callanan DG, Jaffe GJ, Martin DF, Pearson PA, Comstock TL. Treatment of posterior uveitis with a fluocinolone acetonide implant: threeyear clinical trial results. Arch Ophthalmol. 2008;126(9):1191-1201.

9. Friedman DS, Holbrook JT, Ansari H, et al. Risk of elevated intraocular pressure and glaucoma in patients with uveitis: results of the multicenter uveitis steroid treatment trial. Ophthalmology. 2013;120(8): 1571-1579.

10. Maleki A, Swan RT, Lasave AF, Ma L, Foster CS. Selective laser trabeculoplasty in controlled uveitis with steroid-induced glaucoma. Ophthalmology. 2016;123(12):2630-2632.

11. Zivney M, Lin P, Edmunds B, Parikh M, Takusagawa H, Tehrani S. Combined glaucoma tube shunt (Ahmed) and fluocinolone acetonide (Retisert ${ }^{\mathrm{TM}}$ ) implantation compared to Ahmed alone in uveitic glaucoma. Ophthalmol Ther. 2016;5(2):223-233.

12. Moore DB, Stinnett S, Jaffe GJ, Asrani S. Improved surgical success of combined glaucoma tube shunt and Retisert $\left({ }^{\circledR}\right)$ implantation in uveitic eyes: a retrospective study. Ophthalmol Ther. 2015;4(2):103-113.

13. Hennein L, Hou J, Stewart JM, et al. Comparison of surgical outcome after Ahmed valve implantation for patients with and without fluocinolone intravitreal implant (Retisert). J Glaucoma. 2016;25(9): e772-e776.
Clinical Ophthalmology

\section{Publish your work in this journal}

Clinical Ophthalmology is an international, peer-reviewed journal covering all subspecialties within ophthalmology. Key topics include: Optometry; Visual science; Pharmacology and drug therapy in eye diseases; Basic Sciences; Primary and Secondary eye care; Patient Safety and Quality of Care Improvements. This journal is indexed on

Submit your manuscript here: http://www.dovepress.com/clinical-ophthalmology-journal

\section{Dovepress}

PubMed Central and CAS, and is the official journal of The Society of Clinical Ophthalmology (SCO). The manuscript management system is completely online and includes a very quick and fair peer-review system, which is all easy to use. Visit http://www.dovepress.com/ testimonials.php to read real quotes from published authors. 